\title{
Facilitating repertoires of coordination, opposition distinction, and comparison in young children with autism
}

\author{
Sarah Dunne, Mairéad Foody*, Yvonne Barnes-Holmes, Dermot Barnes-Holmes, and Carol Murphy \\ Department of Psychology, National University of Ireland Maynooth
}

\begin{abstract}
According to Relational Frame Theory (RFT), repertoires of derived relational responding are essential for the development of human verbal behavior. As a result, the implications of relational framing for the education of developmentally disabled populations may be immense. Although at the level of process, there appears to be little difference among specific relational frames, there is potentially a natural sequence to their emergence in typical development. However, there is very little published evidence of training children across multiple frames consecutively. The current research comprised four studies that explored an extended sequence of training and testing in the relational frames of coordination, opposition, distinction, and comparison in a sample of nine young children with autism. The results demonstrate the relative ease with which relational deficits in these areas were remediated. In addition, the relationship between outcomes on the Verbal Behavior Milestones and Placement Program-Assessment (VB-MAPP) and individual relational training requirements was investigated.
\end{abstract}

KEYWORDS: coordination, distinction, opposition, and comparative relations

$\mathrm{R}$ ESEARCHERS WORKING UNDER THE rubric of relational frame theory (RFT) have argued that derived relational responding is at the core of complex human verbal behavior, such as humor, story-telling, perspective-taking, and deception (e.g., Barnes-Holmes, McHugh, \& Barnes Holmes, 2004; Hayes, Barnes-Holmes, \& Roche, 2001). Specifically, RFT relies heavily on the concepts of arbitrarily applicable relational responding (A A RR) and multiple stimulus relations (MSR) in its attempts to provide a contextual, functional, behavioral account of these complex cognitive skills. Indeed, these two concepts distinguish RFT from other behavioral accounts of stimulus relations (e.g., Sidman's equivalence, 1974) and have permitted exploration of areas not previously believed to be within the remit of behavior analysis.

\section{» GENERATING REPERTOIRES OF AARR}

For RFT, naming stimuli or events is at the heart of the development of verbal behavior. In naturalistic settings, there are many instances of naming behavior that are directly reinforced. For example, imagine that a parent holds up a child's favorite toy and says "Who's this?" If the child emits the correct name (e.g., "Teddy"), the parent will likely say "Well done". We might refer to this as an explicitly trained object-name relation. On another occasion, the parent may explicitly establish a reverse name-object relation. For example, imagine that the parent says "Where's Dolly?" and the child points to the correct toy (i.e., the doll), and again the parent

\footnotetext{
*Corresponding Author: Mairéad Foody - Mairead.Foody@nuim.ie
}

says "Well done". Consider, however, a third type of interaction that may result from the two types illustrated above. Imagine, on this occasion, that the parent says "Where's Teddy?" and the child points to the teddy, even though this precise interaction (i.e., hear Teddy's name-point to Teddy) has never been explicitly reinforced. That is, the child may have experienced direct training with object-name relations involving the teddy and name-object relations involving the doll, but never name-object relations involving the teddy. For RFT, the child's novel but successful performance on the third type of interaction is an example of a derived mutually entailed name-object coordination relation, that is based upon the history of explicitly trained alternative object-name and name-object coordination relations, which is then applied to a novel name-object relation context (in this case with the teddy). In these cases, the novel or emergent behavior is truly derived (because it has no history of direct reinforcement), but is based upon a history of direct training with similar stimuli and relations. Of course, the concept of mutually entailed coordination relations is synonymous with the more traditional concept of symmetry, at least when coordination relations are involved. For RFT, natural language training involves an almost infinite number of trained and derived exemplars involving words and their related objects.

Mutually entailed relations, including coordination relations, can be readily trained in individuals in whom these repertoires are found to be absent or deficient. And according to RFT, multiple exemplar training (MET) is an effective tool for establishing these skills. In short, MET involves explicit testing and training in 
exemplars of a target relational skill. Consider the experimental trials used by Barnes-Holmes, Barnes-Holmes, Roche, and Smeets (2001A) in their research with 16 children, aged 4-5 years old. Training comprised two simple action-object discriminations that involved selecting A1 (a car) in the presence of the experimenter waving (i.e., training wave-A1), and selecting A 2 (a doll) in the presence of the experimenter clapping (clap-A2). These selections were reinforced to criterion. A subsequent test focused on the derived mutually entailed object-action relations (A1-wave and A2-clap). If the children failed the test, they received explicit object-action training on the same set (i.e., A1-wave and A2- clap) to criterion. This exemplar training continued across multiple stimulus sets until all children demonstrated derived mutually entailed relations on a novel set. Similar findings have been reported by Barnes-Holmes, Barnes-Holmes, Roche, and Smeets (2001B), and Gómez, López, Martín, Barnes-Holmes, and Barnes-Holmes (2007).

For RFT, of course, verbal behavior comprises much more than the derivation of mutually entailed coordination relations between objects and words. According to the theory, the derivation of combinatorially entailed relations is the next step up in complexity, but is still present very early on in natural language training. Consider the following example. Imagine that a parent holds up a child's favorite doll and asks "What is Jane"? If the child emits the correct name (e.g., "doll"), the parent will likely say "Well done". On another occasion, the parent may hold up the doll and ask "What is a doll?" If the child emits the correct name (e.g., "toy"), the parent will again likely say "Well done". Now imagine the parent asks the child "what is Jane?" and the child responds correctly with "toy". For RFT, the emergent relation between "toy" and "doll" is a derived combinatorially entailed coordination relation. As before, the child may have received explicit training for name-doll and doll-toy relations, but even without explicit training, a specific name-toy relation will be derived. This derived response, of course, is synonymous with the more traditional concept of transitivity. Several RFT studies have successfully used MET to facilitate other combinatorially entailed relations (e.g., opposite; Barnes-Holmes, Barnes-Holmes, \& Smeets, 2004).

\section{Coordination}

The examples above all involved relations of coordination or equivalence. And coordination appears to be the most basic frame that infants come into contact with through natural language interactions, and may be the basis on which other frames are established (Lipkins, Hayes, \& Hayes, 1993; Luciano, Gómez-Becerra, \& Rodríguez-Valverde, 2007). This frame requires an individual to respond to contextual cues such as "is" ("is the same as", "equals", etc), which control the derivation of the coordination relations. In simple terms, "is", for example, specifies that two stimuli are arbitrarily coordinated. Consider experimental trials presented by O'Connor, Rafferty, Barnes-Holmes, and Barnes-Holmes (2009) who successfully employed MET to establish coordination relations in 15 children with ASD and three typically-developing children. Participants were trained to establish coordination relations among words, their related objects, and their related pictures, using nameable and familiar stimuli. Training A B relations established mutually entailed relations between the written words (A stim- uli) and pointing to objects (в stimuli). This was followed by в С training (i.e., see objects, point to pictures). The combinatorial entailment (equivalence) tests involved the AC and CA relations (i.e., see word-point to picture; and see picture-point to word). The results demonstrated that MET successfully facilitated equivalence responding on a novel set with six out of eight children. In addition, the findings suggested a relationship between verbal ability and training requirements, such that participants with lower verbal ability required more exposures to explicit training of the target coordination relations. Several other studies have also explored coordination relations. For example, Barnes-Holmes, Barnes-Holmes, Smeets, and Luciano (2004) demonstrated the derived transfer of happy and sad mood functions through coordination relations in a sample of 16 adults. Carr, Wilkinson, Blackman, and McIlvane (2000) also established coordination responding in low-functioning individuals who showed deficits in these repertoires.

\section{Opposition}

The frame of opposition involves arbitrarily applying the relational cue "is the opposite of" or its equivalent along a specific dimension (e.g., hot is the opposite of cold). Consider research by Barnes-Holmes et al. (2004) who successfully employed MET to establish opposite relations in three typically-developing children. The basic experimental trial required the child to select the most valuable coin/s (from four possible options). Consider the following instruction: "Coin A buys many, and A is opposite to B, and B is opposite to C, and C is the opposite to D". Explicit training and increasingly complex testing (e.g., where the coins were presented randomly) continued until participants were responding to trials with 10-coin sequences. Several other studies have investigated this relational frame. For example, Dymond, Roche, Forsyth, Whelan, and Rhoden (2008) demonstrated the derived transfer of avoidance functions in accordance with opposite relations, while Whelan and Barnes-Holmes (2004) demonstrated the transfer of a punishing function.

\section{Distinction}

The relational frame of distinction involves responding to arbitrary differences among stimuli. Like opposition, distinction involves responding along a particular dimension by arbitrarily applying the relational cue "is different from" (e.g., men are different from women). Unlike opposition, however, distinction relations often do not specify the relevant dimension. For example, if you are told that 'men are different from women', it is unclear what these differences. Several RFT studies have investigated distinction relations. For example, Roche and Barnes (1996), and Steele and Hayes (1991) established responding in accordance with distinction relations in teenagers and adults.

\section{Comparison}

The comparative frame involves responding to one event in terms of a quantitative or qualitative relation along a specified dimension with another event. Comparative frames may be divided into specific sub-types, such as bigger-smaller, brighter-darker, etc. The different types are, in part, defined by the dimension along which the relation applies (e.g., size or speed). Comparative frames may 
also involve quantification of the dimension (e.g., ' $A$ is more than B and B is more than $C$ '). Consider experimental trials presented by Barnes-Holmes, Barnes-Holmes, Smeets, Strand, and Friman (2004) who successfully employed MET to establish comparative relations in three typically-developing children. Training A B relations involved selecting the coin (from two possible options) that buy more sweets. Consider the following instruction: "Coin A buys less than coin B, so which coin would you take to buy as many sweets as possible"? Training ВC relations was identical, but now compared coin B with a new coin C. Training ABC relations then involved all three coins. Consider the following instruction: "If coin A buys less than coin $\mathrm{B}$, and if coin B buys less than coin C, which coin would you take to buy as many sweets as possible"? This was followed by an ABC test with novel stimuli. The results demonstrated that MET was a useful way to establish comparative relations and related generalization in young children. Berens and Hayes (2007) reported similar outcomes with typically-developing children.

\section{॥ THE RELATIONAL SEQUENCE}

Although at the level of process, there appears to be little difference between specific relational frames, there is potentially a natural sequence to their emergence in typical development. For example, opposition relations are most likely established, at least to some extent, after the emergence of coordination relations. This is because a coordination relation may be derived from opposition relations. For example, if A is opposite to в and в is opposite to C, then $\mathrm{A}$ and $\mathrm{C}$ are the same. To date, there appears to be no published research that has specifically addressed the optimal sequence for establishing relational frames, although this type of work may have significant educational implications. Indeed, RFT research has already demonstrated a link between derived relational responding and verbal ability as measured by intelligence tests (e.g., Gore, Barnes-Holmes, \& Murphy, 2010; O'Hora, Pelaez, BarnesHolmes, \& Amesty, 2005; O'Toole \& Barnes-Holmes, 2009). For instance, Cassidy, Roche, and Hayes (2011) successfully employed MET to establish a range of relational frames in young children, which subsequently correlated with improved performances on the Wechsler Intelligence Scale for Children-Fourth Edition (WISC-IV). As a result of this, and the studies above that outline the establishment of broad repertoires of relational responding, Luciano et al. (2009) have argued that Applied Behavior Analysis (АВA) intervention programmes should incorporate training and testing in relational frames.

The current research was the first to explore the establishment of an extended sequence of training and testing in consecutive core relational frames in a sample of nine young children with a diagnosis of autism. Specifically, Study 1 sought to establish or facilitate coordination relations. Study 2 sought to establish/facilitate relations of opposition with four of the nine children from Study 1. Study 3 attempted to establish/facilitate distinction relations in two of the previous four children and Study 4 established/facilitated comparison relations in the same two participants. In addition, the putative relationship between training requirements for each relational frame and outcomes on the Verbal Behavior Milestones and Placement Program-Assessment (v B-M A PP; Sundberg, 2008) was investigated.

\section{Study 1: establishing coordination relations}

\section{» METHOD}

\section{Participants}

Nine children, all independently diagnosed with autism, participated in Study 1. There were six males and four females, all aged between 3 years/2months and 5 years/ 1 month. All participants were screened on the VB-MAPP. On this basis, their verbal behavior competence was categorized as follows: two children at Level 1 (lowest level), five at Level 2 (intermediate level), and two at Level 3 (highest level). The characteristics of all children are presented in Table 1.

\section{Setting}

All sessions were conducted in a small quiet classroom in each participant's respective preschool. Only the Researcher and the child were present in the room and were seated side-by-side at a small table. The maximum duration of a session was 2omin., with no more than four sessions per week.

\section{Materials}

The current work involved cue cards for work commencement and ending, as well as reinforcers for correct responding during training and on-task behavior during testing. Study 1 involved 10 sets of picture stimuli, each with three members - one designated

Table 2. Examples of the namable and unnamable stimuli employed in study 1

\begin{tabular}{llll}
\hline Stimulus sets & A & B & C \\
\hline Familiar set & & & \\
Unfamiliar set & & &
\end{tabular}


Table 3. Results of Testing and Training for Coordination Responding for Stages 1-2

\begin{tabular}{|c|c|c|c|c|c|c|c|c|c|c|c|c|}
\hline \multirow[b]{2}{*}{$\mathbf{P}$} & \multicolumn{6}{|c|}{ Stage 1: testing \& training familiar stimuli } & \multicolumn{6}{|c|}{ Stage 2: testing \& training unfamiliar stimuli } \\
\hline & AB train. & $\mathrm{AB}$ test $(20)$ & $\mathrm{BC}$ train. & $\mathrm{BC}$ test (20) & Symm. (40) & Equiv (40) & AB train. & AB test $(20)$ & $\mathrm{BC}$ train. & $\mathrm{BC}$ test (20) & Symm. (40) & Equiv. (40) \\
\hline 1 & 80 & 20 & 118 & 20 & 40 & 21 & 79 & 20 & 80 & 20 & 40 & 39 \\
\hline \multirow[t]{2}{*}{2} & 80 & 20 & 103 & 20 & 36 & 35 & \multirow{2}{*}{79} & \multirow{2}{*}{20} & \multirow{2}{*}{80} & \multirow{2}{*}{20} & \multirow{2}{*}{40} & \multirow{2}{*}{40} \\
\hline & $80^{*}$ & 20 & 80 & 20 & 40 & 40 & & & & & & \\
\hline 3 & 80 & 20 & 80 & 19 & 40 & 40 & 78 & 19 & 80 & 20 & 38 & 39 \\
\hline 4 & 117 & 20 & 120 & 19 & 40 & 40 & 80 & 20 & 80 & 20 & 40 & 40 \\
\hline \multirow[t]{2}{*}{5} & 140 & 16 & 78 & 20 & 31 & - & 192 & 18 & 111 & 20 & 27 & - \\
\hline & 78 & 19 & 85 & 19 & 38 & 38 & $111^{*}$ & 20 & 80 & 20 & 40 & 39 \\
\hline 7 & 78 & 20 & 97 & 20 & 40 & 40 & J & 20 & 0 & $2 v$ & & 70 \\
\hline 8 & 96 & 19 & 77 & 20 & 40 & 40 & 79 & 20 & 79 & 20 & 40 & 40 \\
\hline 9 & 80 & 20 & 80 & 20 & 40 & 40 & 80 & 20 & 80 & 20 & 40 & 39 \\
\hline
\end{tabular}

Note. *Indicates the presentation of a novel set. - Indicates no test was provided

as stimulus A (e.g., A1), one designated as B (e.g., B1), and one designated as C (e.g., C1). Hence, each set comprised of a three-member class (e.g., A1-B1-C1). All of the stimuli that comprised Sets 1-6 were nameable and familiar pictures (e.g., picture of a car). In contrast, all of the stimuli in Sets 7-10 were unnameable and unfamiliar drawings (e.g., picture of the Greek symbol beta). One example of a familiar and unfamiliar stimulus set is illustrated in Table 2 (the full list of stimuli may be obtained from the authors).

\section{Programmed consequences}

A correct response consisted of a participant emitting a vocal response that corresponded to the correct answer of the question. When this occurred, the Researcher provided social praise and access to the specified reinforcer (e.g., "Yes that is correct, well done, here is a sweet"). An incorrect answer was defined as any vocal response that did not correspond to the correct answer or no response within 5 s. of the start of a trial. Specific contingencies were always in place for on-task behavior (e.g., verbal praise such as "nice sitting"). This reinforcement always occurred after a delay of $30 s$. from the end of the previous test trial.

\section{Procedure}

Study 1 consisted of two stages with four phases each. Stage 1 was designed to test and train coordination relations using familiar stimuli. Phase 1 of Stage 1 involved training and testing A B relations and Phase 2 involved $\mathrm{BC}$ relations. Phase 3 involved a test of вA and Св relations, while Phase 4 involved a test of СA and AC relations. Stage 2 consisted of the same four phases with unfamiliar stimuli. If participants failed the test in any stage, they immediately proceeded to training. If participants had been trained on a particular set of stimuli, they completed testing on a novel set before proceeding to the next stage.
Stage 1: Familiar stimuli. Phase 1: AB training and testing. Phase 1 involved explicit training of the two target AB relations (e.g., A1-B1 and A2-B2) from each class. At the beginning of each trial, the sample A stimulus (e.g., A1) and two comparison stimuli (B1 and B2) were placed down side-by-side on the table. A correct response involved the participant pointing to, touching, or picking up the appropriate comparison (e.g., selecting $\mathrm{B} 1$ in the presence of A1). Training both AB trial-types was identical and each was presented in separate blocks of 10 trials, with the locations of the comparisons counterbalanced. Training commenced with A1-B1 and this continued until participants emitted $9 / 10$ consecutively correct responses (90\% accuracy). Training A 2-B2 then began and continued until the same criterion was reached. Once criterion was reached on A2-B2, participants were presented with a test block of 40 mixed trials, 20 A1-B1 and 20 A 2-B2.

Phase 2: $B C$ training and testing. Participants who passed the $A B$ test proceeded immediately to $\mathrm{BC}$ training and testing. This was identical to Phase 1, but involved вC relations (B1-C1 and B2-C2).

Phase 3: Testing $B A$ and $C B$ relations. Participants who passed the BC test proceeded immediately to mutual entailment (symmetry) testing in Phase 3. Each test comprised of 40 mixed trials: 10 B1$\mathrm{A} 1,10 \mathrm{~B} 2-\mathrm{A} 2,10 \mathrm{C} 1-\mathrm{B} 1$, and $10 \mathrm{C} 2-\mathrm{B} 2$. The accuracy criterion was 90\% per block. Participants who passed proceeded to the combinatorial entailment (equivalence) test in Phase 4. Alternatively, participants who failed repeated the conditional discrimination training in Phases 1 and 2, but with two novel stimulus sets (i.e., Sets 3 and 4; $\mathrm{A} 3-\mathrm{B} 3$ and $\mathrm{A} 4-\mathrm{B} 4)$.

Phase 4: $\mathrm{CA}$ and $\mathrm{AC}$ equivalence testing. Phase 4 was identical to Phase 3 , but involved a mixed 40-trial block of $10 \mathrm{C} 1-\mathrm{A} 1,10 \mathrm{C} 2-\mathrm{A} 2$, 10 A1-C1 and 10 A2-C2 trials. Participants who passed proceeded to Stage 2. Participants who failed returned to Phases 1 and 2, but again with two novel sets (e.g., Sets 5 and 6; A5-B5 and A6-B6). 
Stage 2: Training and testing unfamiliar stimuli. Once participants demonstrated equivalence in Phase 4 of Stage 1, they were exposed to the same four phases again, but with unfamiliar stimuli.

\section{" RESULTS}

All nine children completed both stages, hence deriving combinatorially entailed relations with both the familiar and unfamiliar stimulus sets. However, the children differed somewhat in terms of the number of training trials and the number of exemplars needed to complete the phases. The number of training trials and exemplars per phase in each stage, as well as the test performances for each participant, are presented in Table 3.

The minimum number of training trials to meet criterion on the A B conditional discriminations in Stage 1 was 40. All children required between 79 and 140 trials (on the first two stimulus sets) before proceeding to the $\mathrm{AB}$ test, which all passed immediately. The minimum number of training trials to meet criterion on the вC conditional discriminations in Stage 1 was 40. All children again required $77^{-129}$ trials, and passed the $\mathrm{BC}$ test immediately. Three of the nine children (Ps 4, 8, and 9) passed the symmetry test immediately, and immediately passed the equivalence test. Two children ( $\mathrm{P} 1$ and $\mathrm{P} 2$ ) passed the first symmetry test, but failed the equivalence test. They were reexposed to the conditional discrimination training (160 trials) on novel sets, passed the $A B$ and $\mathrm{BC}$ tests, passed the second symmetry test, and passed the second equivalence test. Four children (PS 3, 5, 6, and 7) failed the first symmetry test with accuracies from $28-32 / 40$. The latter were then reexposed to the conditional discrimination training using two novel sets.

Three participants (Ps 3, 5, and 7) completed the conditional discrimination training in a total of 158-175 trials and passed the $\mathrm{AB}$ and $\mathrm{BC}$ tests immediately. They also passed the (second) symmetry test immediately and all except $\mathrm{P} 3$ also passed the subsequent equivalence test. Participant 3 was reexposed to the conditional discrimination training on the fifth and sixth sets $(160$ training trials). He passed both А $\mathrm{B}$ and $\mathrm{BC}$ tests, passed the third symmetry test, and passed the second equivalence test.

The remaining P 6 required 170 trials to complete the second series of conditional discrimination training. Although she passed the А В and вС tests immediately, she again failed the second symmetry test (23/40). She required 158 trials to complete the third series of conditional discrimination training and passed the $A B$ and BC tests immediately. She passed the (third) symmetry test and the subsequent equivalence test immediately.

All nine children completed the A B conditional discrimination training in Stage 2 in 78-192 trials, and all passed the AB test immediately. They required 77-111 training trials on the $\mathrm{BC}$ relations, and passed the BC test immediately. All (except P5) passed the symmetry and equivalence tests immediately. Participant 5 failed the first equivalence test and was reexposed to training on a novel set. She required 191 trials to complete the second set of conditional discrimination training and passed the symmetry and equivalence tests immediately.

The relationship between participants' VB-MAPP scores and the amount of conditional discrimination training required to reach criterion was explored using the Pearson
Table 4. Example of the Big/Small Dimension Targeted in Study 2

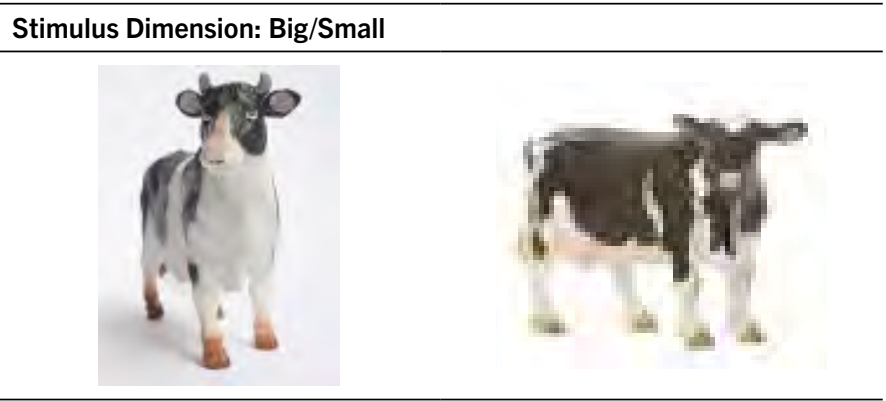

product-moment correlation coefficient. There was a strong negative correlation between the two variables $(r=-.525)$, with higher scores on the VB-MAPP associated with lower levels of training. However, due to the small sample size this did not reach statistical significance $(p=.15)$.

\section{Study 2: establishing opposition relations " METHOD \\ Participants, settings and materials}

Four of the nine children from Study 1 participated in the current study (Ps 4, 5, 7, and 9). All aspects of the setting were identical to the previous study. In addition the study involved several pairs of, and individual, nameable and familiar colored picture stimuli. Specifically, Stage 1 involved 10 familiar colored picture stimuli (e.g., a dog), while Stages 2-4 primarily involved 10 alternative pairs of nameable and familiar stimuli (see Table 4 for examples). Each pair contained two pictures of the same item, which differed along one of ten physical dimensions. For example, one pair of pictures contained a large cow vs. a small cow, while another pair depicted a happy face vs. a sad face. The specific dimensions targeted were: big/small; long/short; wet/dry; hot/cold; happy/sad; clean/dirty; empty/full; dark/light; rough/smooth; and heavy/light. The procedure in Stages 2-4 primarily involved only 10 picture pairs (one per dimension), but additional pairs of pictures depicting the same dimension, but with different items, were available when necessary. Finally, the arbitrary opposition test in Stage 5 involved 8 pairs of identical pictures that were not used in the previous stages. The full list of stimuli may be obtained from the authors.

\section{Procedure}

The programmed consequences were identical to Study 1. Study 2 consisted of testing and training oppositional responding from the basic skill of Y ES/NO responding through to establishing arbitrary opposite relations across five stages. In all stages, if participants failed any of the tests they proceeded immediately to training on that set. If participants had been trained on a particular set of stimuli, the next testing phase always commenced with a novel set.

Stage 1: Testing Y ES/NO responding. Stage 1 comprised of a 30-trial test of YES/NO responding using the 10 familiar pictures (e.g., a cow, see Table 4). At the beginning of each trial, the Researcher held up one picture and asked "Is it a (correct/incorrect name of item?)" The first block of 10 trials all required y Es responding (because the correct item in the picture was specified in the question). 
Table 5. Results of Testing and Training Opposition Responding for Stages 1-5

\begin{tabular}{|c|c|c|c|c|c|}
\hline $\mathbf{P}$ & $\begin{array}{l}\text { Stage 1: YES/NO } \\
\text { responding test (30) }\end{array}$ & $\begin{array}{c}\text { Stage 2: identifying } \\
\text { nonarbitrary dimensions } \\
\text { testing/training }(100)\end{array}$ & $\begin{array}{l}\text { Stage 3: opposite big/ } \\
\text { small test (20) }\end{array}$ & $\begin{array}{c}\text { Stage 4: nonarbitrary } \\
\text { opposite test (120) }\end{array}$ & $\begin{array}{l}\text { Stage 5: arbitrary } \\
\text { opposite test ( } 80)\end{array}$ \\
\hline 5 & 30 & $\begin{array}{c}95 \\
20 T \text { rough/smooth } \\
100^{*}\end{array}$ & $\begin{array}{c}16 \\
80 T \\
20\end{array}$ & 114 & $\begin{array}{c}72 \\
\text { 240T big/small } \\
76^{\star}\end{array}$ \\
\hline 7 & 30 & $\begin{array}{c}90 \\
\text { 10T long/short } \\
100^{*}\end{array}$ & $\begin{array}{c}10 \\
40 T \\
19\end{array}$ & 118 & 71 \\
\hline 4 & 30 & 100 & $\begin{array}{c}10 \\
40 T \\
20^{*}\end{array}$ & 120 & 80 \\
\hline 9 & 30 & $\begin{array}{c}96 \\
\text { 10T long/short } \\
100^{*}\end{array}$ & 20 & 120 & 80 \\
\hline
\end{tabular}

Note. *Indicates testing on a novel set for the specified dimension

The second block of 10 trials all required no responding (because an incorrect item was specified in the question). The third block of 10 trials was mixed randomly, with five YEs trials and five NO trials. The mastery criterion was $27 / 30$. When the children reached criterion they proceeded directly to the next stage.

Stage 2: Identifying nonarbitrary dimensions. Stage 2 involved the 10 pairs of pictures. At the beginning of each trial, one pair was placed side by side on the table and participants were asked, for example, "Show me the small one". In order to respond correctly, they were required to select the stimulus that matched the specified nonarbitrary dimension. Stage 2 always commenced with a 10-trial test block that targeted only the big/small dimension (i.e., 5 big trials and 5 small trials). The mastery criterion was $90 \%$ accuracy (9/10). Participants who achieved this proceeded immediately to a subsequent 10-trial test block targeting the second nonarbitrary dimension (i.e., long/short). Participants who failed the initial big/ small test received explicit training on this dimension using the same set of pictures until they achieved 9/10 consecutively correct responses. They were then immediately exposed to a second big/ small test involving a novel set of pictures. This pattern of testing and training each dimension continued until competence on all 10 nonarbitrary dimensions was demonstrated.

Stage 3: Testing and training opposite relations with big/small. Stage 3 involved the testing and training (if necessary) opposite relations using only the big/small dimension. The stage commenced with a 20-trial test block in which participants were required to select the opposite dimension in 10 trials (e.g., "Show me the opposite of the big one") and name the opposite dimension in the remaining 10 trials (e.g., Researcher pointed to the small stimulus and said "This one is small".) The mastery criterion was $18 / 20$ and participants who passed proceeded to the next stage. Participants who failed received explicit training in blocks of 20 trials until they reached criterion on the same set of pictures. They were then reexposed to the test using a novel set of big/small pictures.

Stage 4: Testing and training nonarbitrary opposite relations. Stage 4 commenced with a combined test of Stages 1-3 involving all 10 dimensions. The test comprised of 120 mixed trials, with a 12-trial block for each dimension. Each 12-trial block comprised four questions from each of the three stages in a fixed sequence.
Consider the example of rough vs. smooth. In the first four trials, the Researcher tested YEs/NO responding (e.g., "Is this one rough? Is this one smooth?") In the next four trials, participants were required to abstract the nonarbitrary dimension (e.g., "Show me the smooth one"). The final four trials tested the opposite relations (e.g., "Show me the opposite of smooth"). The mastery criterion was 110/120 and participants who passed proceeded to the next stage.

Stage 5: Testing and training arbitrary opposite relations. Stage 5 was identical to Stage 4, except that all of the target relations were arbitrary. That is, although the 10 stimulus dimensions targeted above were referred to, the stimuli in question were actually physically identical. The test comprised 80 mixed trials, with an 8-trial block for each arbitrary dimension. Each 8-trial block comprised four questions each from Stages 2 and 3 in a fixed sequence. Consider again the example of rough vs. smooth. In the first four trials, participants were required to abstract the nonarbitrary dimension (e.g., the Researcher pointed to one stimulus and said "If this one is smooth, show me the smooth one"). The next four trials tested the opposite relations (e.g., "If this one is rough, show me the opposite of rough"). The mastery criterion was $7 / 8$ correct responses. Participants who failed were exposed to explicit training to criterion and were then reexposed to the test involving 10 new stimulus sets, one for each dimension.

\section{" RESULTS}

All four children completed all five stages, but differed in the training needed to do so. The number of training trials and exemplars per stage and the test performances for each participant are presented in Table 5.

All four children passed the YES-NO test in Stage 1 with perfect performances. Only $\mathrm{P} 4$ correctly identified all nonarbitrary dimensions in the Stage 2 test, but failed the big/small test in Stage 3. She reached criterion in 40 training trials ( 2 blocks of 20 ) and passed the test with perfect responding on a new set. She then passed Stages 4 and 5 with perfect performances. Participant 9 failed on the long/short dimension in Stage 2 and reached criterion in the minimum 10 training trials. He then passed a second test block on long/short trials with a new set, and passed Stages 3, 4, and 5 with perfect responding. 
Participant 5 failed on the rough/smooth dimension in Stage 2, reached criterion in 20 training trials, and then passed a second test block on rough/smooth trials with a new set. However, she then failed the test in Stage 3 and required 80 training trials to reach criterion (i.e., 4 blocks of 20). She then passed a second test on a novel set with perfect responding. This child reached criterion on the Stage 4 test, but failed the arbitrary opposition test on the big/small trials in Stage 5. She required 240 training trials (i.e., 30 blocks of 8 ) to reach criterion on this dimension before passing the test with a novel set. She then passed the test trials on all remaining dimensions.

Participant 7 failed on the long/short dimension in Stage 2, reached criterion in 10 training trials, and then passed a second test block on long/short trials with a new set. However, he then failed the test in Stage 3 and required 40 training trials to reach criterion. He then passed a second test on a novel set. This child then passed the tests in Stages 4 and 5.

The relationship between participants' VB-MAPP scores and the amount of conditional discrimination training required to reach criterion was explored using the Pearson product-moment correlation coefficient. There was a strong negative correlation between the two variables $(r=-.683)$, with higher scores on the VB-MAPP associated with lower levels of training. However, due to the small sample size $(N=4)$, this did not reach statistical significance $(p=.32)$.

\section{Study 3: establishing distinction relations " METHOD}

Participants, setting, and materials

Two of the four children from Study 2 participated in the current study (Ps 4 and 9). All aspects of the setting were identical to the previous study. In addition, Stage 1 involved 10 pairs of matching colored picture stimuli (e.g., two pigs). Testing and training the abstraction of nonarbitrary dimensions in Stage 2 also involved four sets ( 2 pairs of each object in a set) of familiar objects (see Table 6 for an example). Each pair of objects was identical to one another, but differed from other pairs on one dimension: same/ different colors; same/different length, same/different textures, and same/different shapes. Stage 3 also involved two sets (three objects per set) of identical cups and identical boxes.

\section{Procedure}

The programmed consequences were identical to the previous studies. Study 3 consisted of testing and training distinction responding from simply recognizing same and different pictures through to using this skill with nonarbitrary stimuli.

Stage 1: Testing nonarbitrary same/different relations. Stage 1 involved 30 test trials to assess responding to nonarbitrary same and different relations. At the beginning of each trial, the Researcher placed three picture cards (two identical pictures and one different) on the table and asked: "Show me the pictures that are the same/different". The first block of 10 trials required participants to select the two identical pictures, while the second block required the selection of the different picture. The third block of trials was randomly mixed, with five same trials and five different trials. The mastery criterion was $27 / 30$.
Table 6. Example of the same/different shape dimension employed in study 3

Nonarbitrary stimulus sets (same/different shapes)

Stage 2: Testing nonarbitrary same/different relations across dimensions. Stage 2 comprised 32 test trials, with four blocks of eight trials for each of the four target stimulus dimensions (i.e., color, length, texture, and shape). During each block, either two stimuli that were identical on the target dimension (e.g., two red bricks) or that differed on the target dimension (e.g., a red brick and a yellow brick) were presented. Participants were asked, for example, "Are the bricks the same/different color?" The format was identical for assessing all four dimensions and the mastery criterion was $28 / 32$.

Stage 3: Testing mutually entailed same/different relations. Stage 3 comprised a 12-trial test block, with six same trials and six different trials. During the same trials, the Researcher presented two identical stimuli and stated, for example, "Box A (pointing to one stimulus) is the same as Box в (pointing to the other stimulus)." During three of the trials, she then asked "Are they the same", while on the three remaining trials, she asked "Are they different?" During the different trials, the Researcher presented two non-identical stimuli and stated, for example, "Box A is different from Box в." Again, during three trials, she asked "Are they the same?" while on the three remaining trials, she asked "Are they different?" The mastery criterion was $100 \%$ because on half of the same trials and half of the different trials, correct responding may have been based on nonarbitrary, rather than arbitrary relations (e.g., when presented with identical stimuli and asked "Are they the same?").

Stage 4: Testing combinatorially entailed same/different relations. Stage 4 comprised a block of 12 test trials. On each trial, three identical objects (i.e., identical cups; labeled A1, B1, and C1) were placed side by side on the table. There were four trials for each of three trial types, referred to as: A same B, B same C, A same/ different C; A same B, B different C, a same/different C; A different B, B same C, A same/different C. On two trials from each set of four, the question involved a same relation (e.g., A same C), whereas on the remaining two trials, the question involved a different relation (e.g., A different C). All trials assessed arbitrary responding to the

Table 7. Results of testing and training distinction responding for stages 1-4

\begin{tabular}{|c|c|c|c|c|}
\hline $\mathbf{P}$ & $\begin{array}{l}\text { Stage 1: same/ } \\
\text { different } \\
\text { test }(30)\end{array}$ & $\begin{array}{l}\text { Stage 2: } \\
\text { nonarbitrary } \\
\text { dimension } \\
\text { test (32) }\end{array}$ & $\begin{array}{l}\text { Stage 3: } \\
\text { mutually } \\
\text { entailed } \\
\text { same/ } \\
\text { different } \\
\text { test (12) }\end{array}$ & $\begin{array}{l}\text { Stage 4: } \\
\text { combinatorially } \\
\text { entailed same/ } \\
\text { different } \\
\text { test (12) }\end{array}$ \\
\hline 4 & 30 & 31 & 12 & $\begin{array}{l}8 \\
240 \mathrm{~T} \\
11^{*}\end{array}$ \\
\hline 9 & 30 & 32 & 12 & 12 \\
\hline
\end{tabular}

Note. *Indicates testing on a novel set 
Table 8. Examples of stimulus sets employed in study 4

Stimulus type

Arbitrary stimuli

combinatorially entailed Ac relation. The accuracy criterion was 11/12. If participants failed the test, they were exposed to explicit training of the target relations and then exposed to the same test with a novel set.

\section{1) RESULTS}

Table 7 presents the test and training performances for each child across the four stages.

Participant 9 passed all tests and required no training at any stage. Participant 4 passed all tests, except Stage 4. Following extensive training (240 trials), she reached criterion and passed a subsequent test with novel stimuli. The relationship between participants' VB-MAPP scores and the amount of conditional discrimination training required to reach criterion was not explored because both participants were categorized at Level 3 on the VB-MAPP.

Table 9. Results of testing and training comparison responding for stages 1-4

\begin{tabular}{ccccc}
\hline $\begin{array}{c}\text { Stage 1: } \\
\text { nonarbitrary } \\
\text { more-than/ } \\
\text { less-than } \\
\text { relations } \\
\text { test (12) }\end{array}$ & $\begin{array}{c}\text { Stage 2: } \\
\text { nonarbitrary } \\
\text { combinatorially } \\
\text { entailed more- } \\
\text { than/less-than } \\
\text { test (24) }\end{array}$ & $\begin{array}{c}\text { Stage 3: } \\
\text { arbitrary } \\
\text { more-than/ } \\
\text { less-than } \\
\text { test (24) }\end{array}$ & $\begin{array}{c}\text { Stage 4: } \\
\text { arbitrary } \\
\text { combinatorially } \\
\text { entailled } \\
\text { more-than/less } \\
\text { than test (24) }\end{array}$ \\
\hline & & & & 8 \\
4 & 11 & 10 & $168 \mathrm{~T}$ \\
& & $312 \mathrm{~T}$ & $24 \mathrm{~T}$ & $18^{*}$ \\
& & $24^{*}$ & $23^{*}$ & $96 \mathrm{~T}$ \\
& & 15 & & $22^{*}$ \\
\hline & & $168 \mathrm{~T}$ & 24 & 23 \\
\hline
\end{tabular}

Note. *Indicates testing on a novel set

\section{Study 4: establishing comparative relations}

\section{" METHOD}

Participants, setting, and materials

The same two children from Study 3 participated again. All aspects of the setting were identical to the previous study. In addition, Study 3 involved sets of identical objects and sets of non-identical coin-shaped objects (see Table 8).

\section{Procedure}

Stage 1: Testing nonarbitrary more-than/less-than relations. Stage 1 involved a fixed block of 12 test trials, first with six more-than relations and then six less-than relations. On each trial, an array of identical items was presented as two stimuli, with one always containing more items than the other (e.g., 10 candies on one side and 3 candies on the other). On the six more-than trials, the Researcher asked "Which has more" and a correct response involved pointing to the stimulus with the larger number of items. On the six less-than trials, the Researcher asked "Which has less" and a correct response involved pointing to the stimulus with the less number of items. The mastery criterion was 90\%.

Stage 2: Testing nonarbitrary combinatorial more-than/less-than relations. Stage 2 involved a block of 24 test trials, with 12 trials for each of two trial types denoted as $\mathrm{A}<\mathrm{B}<\mathrm{C}$ and $\mathrm{A}>\mathrm{B}>\mathrm{C}$, always involving an array of three sets of items. Similar to Stage 1, the three stimuli involved different numbers of items (e.g., 10 candies on one side, 5 candies in the middle, and 3 candies on the other side). On all trials, participants were asked about the combinatorially entailed AC relation (i.e. "Is A more than or less than C"). On the six A $<\mathrm{B}<\mathrm{C}$ trials, a correct response involved deriving $\mathrm{A}<\mathrm{C}$, while on the six A $>\mathrm{B}>\mathrm{C}$ trials, a correct response involved deriving A $>$ C. The mastery criterion was 22/24. Participants who failed were exposed to explicit training of the target relations and were then tested on a novel set.

Stage 3: Testing arbitrary more-than/less-than relations. Stage 3 involved a fixed block of 24 test trials, with six trials for each of four trial types denoted as $\mathrm{A}>\mathrm{B}$; $\mathrm{B}<\mathrm{A}$; $\mathrm{B}>\mathrm{A}$; and $\mathrm{A}<\mathrm{B}$. On each trial, two identical items (referred to as 'coins') were presented. On three trials for each trial type, the Researcher asked "Which has more" and a correct response involved pointing to the stimulus which had been arbitrarily identified as worth more (e.g., selecting A when presented with A > B). On the other three trials for each trial type, the Researcher asked "Which has less" and a correct response involved pointing to the stimulus which had been arbitrarily identified as worth less (e.g., selecting в when presented with $\mathrm{A}>\mathrm{B}$ ). The mastery criterion was $90 \%$. Participants who failed were exposed to explicit training of the target relations and were then tested on a novel set.

Stage 4: Testing arbitrary combinatorial more-than/less-than relations. Stage 4 involved a block of 24 test trials, with 12 trials for each of two trial types denoted as $\mathrm{A}<\mathrm{B}<\mathrm{C}$ and $\mathrm{A}>\mathrm{B}>\mathrm{C}$, always involving an array of three sets of identical coins. On all trials, participants were asked about the combinatorially entailed AC relation (i.e., "Is A more than or less than C"). On the six $\mathrm{A}<\mathrm{B}<\mathrm{C}$ trials, a correct response involved deriving $\mathrm{A}<\mathrm{C}$, while on the six A $>$ B $>$ C trials, a correct response involved deriving 
A > C. The mastery criterion was 90\%. Participants who failed were exposed to explicit training of the target relations and were then tested on a novel set.

\section{" RESULTS}

The results for each participant according to each stage are presented in Table 9.

Participant 9 passed Stage 1 with a perfect score, but required 168 training trials to reach criterion. The child then passed the test using a novel set. He thereafter passed Stages 3 and 4 immediately (24/24 and 23/24, respectively). Participant 4 also passed Stage 1 with a perfect score, but similarly required 312 training trials to reach criterion before passing the test with a perfect score on a novel set. However, she also failed Stage 3 and needed 24 training trials (i.e., one block) before completing the test on the second exposure. This child also failed Stage 4 (8/24) and needed 168 training trials before reaching criterion. However, she also failed the second exposure to the test with the new set. She received an additional 96 training trials and finally passed the third test completing the test $(22 / 24)$.

\section{" DISCUSSION}

The current set of four studies demonstrates the establishment or facilitation of derived relational responding in accordance with coordination, opposition, distinction, and comparative relations in a sample of nine children with autism.

Study 1 was designed to train coordination relations using familiar and unfamiliar stimuli. All nine children differed in terms of the amount of training they required to complete the symmetry and equivalence tests for coordination on the familiar stimuli. That is, three children (Ps 4, 8, and 9) passed both tests with training. Two children (Ps 5 and 7 ) failed the first symmetry test and required a modest amount of training (381-444) to pass the second symmetry test, but both then passed equivalence immediately. Two children (Ps 1 and 2) passed the first symmetry test but failed the first equivalence test, although they passed the second equivalence test after modest training (343-357 trials). One child ( $\mathrm{P} 6$ ) failed two symmetry tests, required extensive training (501 trials) before passing the third test, and passed equivalence immediately. The remaining child ( $\left.\mathrm{P}_{3}\right)$ failed the first symmetry test and required 315 trials before passing the second symmetry test. He subsequently failed equivalence and required 160 trials to pass the second equivalence test. After these patterns of testing and training, eight of the nine children (with the exception of P5) passed the symmetry and equivalence tests with the unfamiliar stimuli in Stage 2 without training.

There appeared to be some overlap between the children's vBMAPP scores and their performances on the coordination relations. Specifically, those categorized at the highest Level 3 (i.e., Ps 4 and 9) required the least training with the familiar stimuli and no training on the unfamiliar stimuli. The five children categorized at the middle Level 2 (i.e., Ps 1, 2, 6, 7, and 8) generally required more (although highly variable amounts of) training with the familiar stimuli and no training on the unfamiliar stimuli. The two remaining children ( $\mathrm{Ps}_{3}$ and 5 ) categorized at Level 1 required the most training on familiar stimuli and one of these children
(P5) also required training with unfamiliar stimuli. These findings support previous research which has shown a relationship between verbal ability and the training requirements and test performances for equivalence with coordination relations (e.g., Barnes, Browne, Smeets, \& Roche, 1995; Barnes, McCullagh, \& Keenan, 1990; Devany, Hayes, \& Nelson, 1986; O'Connor et al., 2009).

All four children in Study 2 of opposition relations passed the YES-No test without training. Only one child (P4) also passed the nonarbitrary dimensions test immediately, but she failed the big/small test and required 40 training trials before passing. She then passed the nonarbitrary and arbitrary opposition tests immediately. Two children (Ps 9 and 7 ) failed the nonarbitrary dimensions test with poor performances on the long/short dimension, and each passed after only 10 trials on that dimension. Subsequently, P9 passed the big/small test, and the nonarbitrary and arbitrary opposition tests without training. In contrast, $\mathrm{P} 7$ required 40 training trials to pass the big/small test, but he then passed both the nonarbitrary and arbitrary opposition tests without training. The remaining $\mathrm{P} 5$ failed the nonarbitrary dimensions test with a poor performance on the rough/smooth dimension, after which 20 training trials were required to pass on this dimension. However, she then required extensive training ( 80 trials) to pass the big/small test. Although she subsequently passed the nonarbitrary opposition test, she failed the arbitrary opposition test (again showing problems on the big/small trials). She required a total of 240 trials on this before passing the full test with a novel set.

Similar to Study 1, there appeared to be some overlap between the children's VB-MAPP scores and their performances on opposition relations as investigated in Study 2. Again, those categorized at the highest Level 3 (i.e., Ps 4 and 9) required the least training overall, and apart from 10 trials on the nonarbitrary dimensions test (P9), and 40 trials on the big/small test ( $(\mathrm{P} 4)$, both children passed all tests without further training. One child categorized at the middle Level $2(\mathrm{P} 7)$ required more training on the nonarbitrary dimensions test (on long/short) and on the big/small test, but was then also successful in passing the remaining tests. The remaining child (P5) was categorized at Level 1 and required the most training in order to pass the nonarbitrary dimensions test (rough/smooth), the big/small test and both opposition tests. Only one previous study has investigated the establishment of opposition relations (Barnes-Holmes et al., 2004), but the trials here differed considerably from that study and the latter did not include alternative scores of verbal ability. However, both studies were similar in that they established opposition responding in children who previously appeared to lack this repertoire.

Study 3 investigated distinction relations. Only two of the original children participated and both had been categorized at VB-MAPP Level 3, hence making it impossible to attempt to explore comparisons on verbal ability. Interestingly, however, some differences were recorded between the participants. One child (p9) passed all tests of distinction responding (i.e., the nonarbitrary same/different test, the nonarbitrary dimensions test, the mutually entailed same/different test, and the combinatorially entailed same/ different test). Although $\mathrm{P} 4$ performed well overall, she required 240 training trials to pass the final combinatorially entailed test. 
In short, P9 performed somewhat better than $\mathrm{P} 4$. To date, there appear to be no published studies of the establishment or facilitation of distinction relations in children.

The same two children participated in Study 4 in the investigation of comparative relations. Again there were considerable differences between the children's performances. Both passed the nonarbitrary more-than/less-than test with two comparisons, but required $168(\mathrm{P} 9)$ or $312(\mathrm{P} 4)$ trials to pass the same test with three comparisons (the combinatorially entailed test). Subsequently, P9 passed the arbitrary more-than/less-than tests with two and with three comparisons without additional training. In contrast, $\mathrm{P} 4$ required 24 training trials to pass the arbitrary two comparisons test and 168 trials to complete the arbitrary three comparisons test. She subsequently failed the second exposure to this test with a novel set. She received an additional 96 training trials and finally passed the third comparative test. Once again, therefore, P9 performed somewhat better than $\mathbf{P} 4$.

Although Ps 4 and 9 had both been categorized as Level 3 on the VB-MAPP, there were notable differences in their performances in Studies 3 and 4. Overall $\mathrm{P} 9$ required considerably less training. As a result, we returned to their original VB-MAPP outcomes and discovered that $\mathrm{P} 9 \mathrm{had}$, in fact, a substantially higher score of 139.5 compared to P4's 93.5. This may, to some extent, account for the differences between training requirements of both children. These findings support previous research which has also shown a relationship between verbal ability and the training requirements and test performances with comparative relations (e.g., Gorham, Barnes-Holmes, Barnes-Holmes, \& Berens, 2009).

It may be argued that the program of training and testing conducted across the four studies facilitated relational responding for some children, but established the target relational repertoires for others. For example, there were a number of areas in which $\mathrm{P} 9$ required no training and the training that was required always involved nonarbitrary relations, which thereafter ensured sound test performances on arbitrary relations. These findings suggest that the child had a broad array of existing relational skills that the training then fostered to facilitate the derived arbitrary performances. In contrast, several other children required explicit training on the arbitrary relations, which appeared to suggest that to some extent the target performances were being established (e.g., P4 required 264 training trials in total to pass the combinatorially entailed comparative test). What is perhaps more important about this is that the testing and training procedures permitted a very precise means of assessing each child's competencies on the target nonarbitrary and arbitrary relations, and training readily resulted in sound demonstrations of arbitrary responding with novel stimuli.

Barnes-Holmes et al. (2004) questioned the optimal training and testing sequence for the establishment of the core relational frames as targeted by the current research. And this raises the interesting (although perhaps simplistic) question about the manner in which these frames are established in the typical developmental trajectory. For example, Barnes-Holmes et al. asked, if the relational frame of sameness facilitates the frame of opposition, which seems likely to some extent because opposition relations incorporate same relations. It is difficult in the current research to address this issue because the sequence of studies was not a between-subjects design and only two children completed the latter two studies. Furthermore, of those two children, both had been assessed at VB-MAPP Level 3. However, the data from P4 does not appear to suggest that the training requirements of this child decreased steadily across the four studies, thus implying that the earlier frames did not greatly facilitate the subsequent frames. However, much more systematic explorations of this issue are needed.

The current research perhaps speaks as much to typical development as to atypical development, but at the very least demonstrates the way in which relational deficits may be tested and trained. None of the children found the procedures tiresome or aversive, and in all cases the target arbitrary performances were demonstrated on tests of novel stimuli in a matter of months. This suggests the feasibility of enhancing existing behavioral intervention programs with RFT testing and training protocols. It is possible, of course, that existing programs directly or indirectly target relational frames and facilitate same through existing training. However, there is no empirical evidence to indicate this and thus the relationship between these two system of intervention represents a potentially important vein of future research.

\section{REFERENCES}

Barnes-Holmes, Y., Barnes-Holmes, D., Roche, B., \& Smeets, P. (2001a). Exemplar training and a derived transformation of function in accordance with symmetry: II. The Psychological Record, 51, 589-604.

Barnes-Holmes, Y., Barnes-Holmes, D., Roche, B., \& Smeets, P. M. (2001b). Exemplar training and a derived transformation of function in accordance with symmetry: I. The Psychological Record, 51, 287-308.

Barnes-Holmes, Y., Barnes-Holmes, D., \& Smeets, P. M. (2004). Establishing relational responding in accordance with opposite as generalized operant behaviour in young children. International Journal of Psychology and Psychological Therapy, 4, 559-586.

Barnes-Holmes, Y., Barnes-Holmes, D., Smeets, P. M., Strand, P., \& Friman, P. (2004). Establishing relational responding in accordance with more-than and less-than as generalized operant behaviour in young children. International Journal of Psychology and Psychological Therapy, 4, 531-558.
Barnes-Holmes, Y., Barnes-Holmes, D., Smeets, P. M., \& Luciano, C. (2004). The derived transfer of mood functions through equivalence relations. The psychological Record, 54, 96-114.

Barnes-Holmes, Y., McHugh, L., \& Barnes-Holmes, D. (2004). Perspective-taking and Theory of Mind: A relational frame account. The Behavior Analyst Today, 5, 15-25.

Barnes, D., Browne, M., Smeets, P., \& Roche, B. (1995). A transfer of functions and a conditional transfer of functions through equivalence relations in three to six year old children. The Psychological Record, 45, 405-430.

Barnes, D., McCullagh, P., \& Keenan, M. (1990). Equivalence class formation in non-hearing impaired children and hearing impaired children. Analysis of Verbal Behavior, 8, 1-11.

Berens, N. M., \& Hayes, S. C. (2007). Arbitrarily applicable comparative relations: Experimental evidence for relational operants. Journal of Applied behaviour Analysis, 40, 45-71. 
Carr, D., Wilkinson, K. M., Blackman, D., \& Mcllvane, W. J. (2000) Equivalence classes in individuals with minimal verbal repertoires. Journal of the Experimental Analysis of Behavior, 74, 101-114.

Cassidy, S., Roche, B., \& Hayes, S. C. (2011). A relational frame training intervention to raise Intelligence Quotients: A pilot study. The Psychological Record, 61, 173-198.

Devany, J. M., Hayes, S.C., \& Nelson, R. O. (1986). Equivalence class formation in language-able and language-disabled children. Journal of the Experimental Analysis of Behavior, 46, 243-257.

Dymond, S., Roche, B., Forsyth, J. P., Whelan, R., \& Rhoden, J. (2008). Derived avoidance learning: Transformation of avoidance response functions in accordance with same and opposite relational frames. The Psychological Record, 58, 269-286.

Gore, N. J., Barnes-Holmes, Y., \& Murphy, G. (2010). The relationship between intellectual functioning and relational perspective-taking. International Journal of Psychology and Psychological Therapy, 10(1), 1-17.

Gorham, M., Barnes-Holmes, Y., Barnes-Holmes, D., \& Berens, N. (2009). Derived comparative and transitive relations in young children with and without autism. The Psychological Record, 59, 221-246.

Gómez, S., López, F., Martín, C. B., Barnes-Holmes, Y., \& Barnes-Holmes, D. (2007). Exemplar training and a derived transformation of functions in accordance with symmetry and equivalence. Psychological Record, 57(2), 273-294.

Hayes, S. C., Barnes-Holmes, D., \& Roche, B. (Eds.). (2001). Relational Frame Theory: A Post-Skinnerian account of human language and cognition. New York: Plenum Press.

Lipkins, G., Hayes, S. C., \& Hayes, L. J. (1993). Longitudinal study of derived stimulus relations in an infant. Journal of the Experimental Analysis of Behavior, 56, 201-239.

Luciano, C., Gómez-Becerra, I., \& Rodríguez-Valverde, M. (2007). The role of multiple-exemplar training and naming in establishing derived equivalence in an infant. Journal of Experimental Analysis of Behavior, 87, 349-365.
Luciano, C., Berens, N.M., Rodríguez, M., Manas, I., Ruiz, F., \& Valdivia-Salas, S. (2009). Acquiring the earliest relational operants: Coordination, Difference, Opposition, Comparison, and Hierarchy. In (Eds.) Y. Barnes-Holmes, \& R. A. Rehfeldt, Derived relational responding: Application for learners with autism and other developmental disabilities. Oakland, CA: New Harbinger.

O'Connor, J., Rafferty, A., Barnes-Holmes, D., \& Barnes-Holmes, Y. (2009). The role of verbal behavior, stimulus nameability and familiarity on the equivalence performances of autistic and normally developing children. The Psychological Record, 59 (10), 53-74.

O'Hora, D., Pelaez, M., Barnes-Holmes, D., \& Amesty, L. (2005). Derived relational responding and human language: Evidence from the WAISIII. The Psychological Record, 58, 155-174.

O'Toole, C., \& Barnes-Holmes, D. (2009). Three chronometric indices of relational responding as predictors of performance on a brief intelligence test: The importance of relational flexibility. The Psychological Record, 59, 119-132.

Roche, B., \& Barnes, D. (1996). Arbitrarily applicable relational responding and human sexual categorization: A critical test of the derived difference relation. The Psychological Record, 46, 451-475.

Sidman, M. (1974). Equivalence relations and behaviour: A research story. Boston, MA: Authors Cooperative.

Steele, D. L., \& Hayes, S. C. (1991). Stimulus equivalence and arbitrarily applicable relational responding. Journal of the Experimental Analysis of Behavior, 55, 519-555.

Sundberg, M. L. (2008). VB-MAPP Verbal behaviour milestones assessment and placement program: A language and social skills assessment program for children with autism or other developmental disabilities. Concord, CA: AVB Press.

Whelan, R., \& Barnes-Holmes, D. (2004). The transformation of consequential functions in accordance with the relational frames of same and opposite. Journal of the Experimental Analysis of Behavior, 82, 177-195. 